\title{
Decomposição de amostras de cerveja com sistema de refluxo para determinação monoelementar por F AAS/AES e determinação multielementar por MIP OES
}

\author{
Beer sample decomposition using a reflux system for the monoelementar determination \\ by F AAS/AES and multielementar determination by MIP OES
}

\author{
Paulo Roberto Pereira de Leão1, Aline Lisbôa Medina ${ }^{1 *}$, Mariana Antunes Vieira', Anderson Schwingel Ribeiro' \\ 1 Universidade Federal de Pelotas (UFPel), Centro de Ciências Químicas, Farmacêuticas e de Alimentos, Laboratório de Metrologia Química (LabMeQui), \\ Capão do Leão/RS - Brasil
}

\section{*Corresponding Author}

Aline Lisbôa Medina, Universidade Federal de Pelotas (UFPel), Centro de Ciências Químicas, Farmacêuticas e de Alimentos, Laboratório de Metrologia Química (LabMeQui), Campus Capão do Leão, CEP: 96160-000, Capão do Leão/RS - Brasil, e-mail: medinaline@gmail.com

Cite as: Beer sample decomposition using a reflux system for the monoelementar determination by $F$ AAS/AES and multielementar determination by MIP OES. Braz. J. Food Technol., v. 21, e2017062, 2018.

\section{Resumo}

Neste trabalho, é descrito o desenvolvimento de um método analítico para análise de amostras de cervejas por técnicas de espectrometria. As amostras foram preparadas por decomposição em meio ácido, em um sistema de refluxo, e as determinações foram realizadas por F AAS (Fe, Mg e Zn), F AES (Na) e MIP OES (Ca, Fe, K, Mg, Mn, Na, Ni e Zn). As melhores condições para a decomposição da amostra foram obtidas através de estudo com delineamento composto


etapa de aquecimento em bloco digestor a $160{ }^{\circ} \mathrm{C}$, por $93 \mathrm{~min}$. Os limites de quantificação do método foram $\leq 0,100 \mathrm{mg} \mathrm{L}^{-1}$ para todos os elementos investigados, independentemente da técnica analítica utilizada, ficando abaixo, por exemplo, dos valores estipulados para Zn e Ni, pela Agência Nacional de Vigilância Sanitária do Brasil. Os estudos comparativos entre as diferentes técnicas avaliadas mostraram, pelo teste T com nível de $90 \%$ de confiança, que não há diferença significativa para as determinações de $\mathrm{Fe}, \mathrm{Mg}$, Na e Zn nas amostras de cerveja. O método de preparo da amostra proposto mostrou ser simples, rápido, de baixo custo e confiável, para análise de cervejas nas diferentes técnicas de espectrometria atômica.

Palavras-chave: Decomposição ácida; Técnicas espectrométricas.

\section{Abstract}

This study describes the development of an analytical method for the analysis of beer samples by atomic spectrometric techniques. The samples were prepared by acid decomposition using a reflux system, and the determinations carried out by F AAS (Fe, Mg and Zn), F AES (Na) and MIP OES (Ca, Fe, K, Mg, Mn, Na, Ni and Zn). The best conditions for sample decomposition were obtained by way of studies using a central rotational compound design: $10.0 \mathrm{~mL}$ of degassed beer followed by the addition of $5.0 \mathrm{~mL}$ of $\mathrm{HNO}_{3}$ and $2.0 \mathrm{~mL} \mathrm{H} \mathrm{O}_{2}$ and a heating step in the digester block at $160{ }^{\circ} \mathrm{C}$ for $93 \mathrm{~min}$. The quantification limits of the method were $\leq 0.100 \mathrm{mg} \mathrm{L}^{-1}$ for all the analytes investigated, regardless of the analytical technique used, values below those established by the Brazilian Health Surveillance Agency for $\mathrm{Zn}$ and $\mathrm{Ni}$, for example. The comparative study of the different techniques showed that, according to the t-test at a 90\% level of confidence, there was no significant difference for the determinations of $\mathrm{Fe}, \mathrm{Mg}, \mathrm{Na}$ and $\mathrm{Zn}$ in the beer samples. The sample preparation method was shown to be simple, fast, low-cost and reliable for the analysis of beers by the different atomic spectrometry techniques.

Keywords: Acid decomposition; Spectrometric techniques. 


\section{Introdução}

A cerveja é uma bebida fermentada a partir de cereais maltados, normalmente de malte de cevada, com ou sem materiais ricos em amido, e aromatizada por lúpulo (AGU, 1995; KEUKELEIRE, 2000). O corpo da cerveja é fornecido pelo malte de cevada, sendo que podem ser utilizados adjuntos ricos em amido, substituindo os ingredientes originais por outros, tais como arroz, milho ou trigo (MARDEGAN et al., 2013). Atualmente, é uma das bebidas mais consumidas no mundo, sendo que, em 2013, - Brasil estava entre os maiores produtores, atrás apenas dos Estados Unidos e da China (CERVBRASIL, 2016). No Brasil, o consumo anual de cerveja é de 47 L/pessoa, enquanto que, para República Checa, Irlanda e Alemanha, maiores consumidores mundiais, o consumo é de 159, 131 e 115 L/pessoa, respectivamente (CNBC, 2017). Estudos indicam que a cerveja pode trazer benefícios à saúde humana quando ingerida de forma moderada, devido à presença de certos componentes, como antioxidantes, fibras, vitaminas e minerais (BAMFORTH, 2002).

Elementos minerais e metálicos encontrados na cerveja podem ser provenientes de fontes naturais (solo, água, cereais, lúpulo e levedura), bem como da contaminação ambiental - fertilizantes e pesticidas -, do processo industrial ou de embalagens (POHL; PRUSISZ, 2007; Ll et al., 1993). Desse modo, o controle desses elementos é de extrema importância, considerando-se que podem interferir nas características da cerveja de maneira desejável ou indesejável, afetando sua produção e podendo, assim, não manter um padrão de qualidade para o produto.

A quantificação de metais em amostras de cerveja, geralmente, é realizada através de técnicas espectrométricas (POHL; PRUSISZ, 2007; ONIANWA et al., 1999; BELLIDO-MILLA et al., 2000; OÑATE-JAÉN et al., 2006; ALCÁZAR et al., 2002, MATSUSHIGE; OLIVEIRA, 1993; PASSAGHE et al., 2015). A espectrometria de emissão atômica com plasma induzido por micro-ondas (MIP OES) é uma nova e interessante alternativa, não só por apresentar características de análise multielementar, o que diminui o tempo da análise e o consumo de amostra, mas também por utilizar um plasma de nitrogênio, que é proveniente de gerador alimentado por ar comprimido, diminuindo, assim, significativamente, os custos das análises.

Para a determinação de metais por técnicas de espectrometria atômica, em que se utiliza a introdução da amostra no equipamento por sistema de nebulização, se faz necessário que a amostra esteja em solução. Embora a cerveja seja uma amostra na forma líquida, sua composição apresenta compostos orgânicos solúveis e frequentemente precipitados em suspensão, o que pode causar interferências (espectrais e não espectrais) nas técnicas de espectrometria atômica, efeito de transporte e obstrução (WIECZOREK et al., 2017; MOORE, 1989). A etapa de preparo da amostra visa eliminar efeitos de matriz e demais problemas, garantindo a exatidão e a eficiência da análise. Uma alternativa para a determinação de metais é o uso de um sistema de refluxo para as decomposições em sistema aberto, o qual evita perdas por volatilização, já que, durante o processo de condensação no dedo frio, a solução formada retém as espécies volatilizadas e, durante o processo de gotejamento, retorna ao meio reacional. Diversas aplicações com esse sistema podem ser encontradas na literatura, como em amostras de arroz (OLIVEIRA et al., 2016; PINHEIRO et al., 2014), goma xantana (ROSA et al., 2016) e amostras biológicas (ORESTE et al., 2013), tecido de ostras (NANO et al., 2009) e vinho (FERREIRA et al., 2009).

Neste trabalho, foi avaliado o uso do sistema de refluxo com dedo frio em sistema aberto para a decomposição de amostras de cerveja, para a posterior determinação de $\mathrm{Fe}, \mathrm{Mg}$ e Zn por F AAS, Na por F AES e Ca, Fe, K, Mg, Mn, $\mathrm{Na}, \mathrm{Ni}$ e $\mathrm{Zn}$ por MIP OES. O objetivo foi desenvolver um método eficiente de preparo de amostra em cerveja que apresentasse baixo custo, segurança para o monitoramento e controle da qualidade do produto final. Entre as amostras avaliadas, estão cervejas de grandes marcas comerciais e cervejas artesanais produzidas no Brasil.

\section{Material e métodos}

\subsection{Reagentes}

Todos os reagentes utilizados foram de grau analítico. Para o preparo das soluções, utilizou-se água deionizada, obtida a partir de um sistema de destilação em um destilador de vidro (MA078/5, Marconi, SP) e, posteriormente, eluída em um deionizador (CS1800 Evolution, Permution, PR). Soluções de trabalho para Mg e Na foram preparadas por diluições feitas a partir de uma solução estoque para AAS contendo $1.000 \mathrm{mg} \mathrm{L}^{-1}$ (Fluka, Buchs, Suíça). Para Zn e Fe, as soluções de trabalho foram obtidas de forma semelhante, a partir de soluções estoque que foram preparadas a uma concentração de $1.000 \mathrm{mg} \mathrm{L}^{-1}$ de Tritisol ${ }^{\circledR}$ (Merck, Darmstadt, Alemanha), em água deionizada. Para Ni, Mn, Ca e K, foi utilizada a solução padrão multielementar para ICP n. ${ }^{\circ} 6$, $100 \mathrm{mg} \mathrm{L}^{-1}$ (Sigma Aldrich, St. Louis, Missouri, EUA). Na etapa de preparo das amostras, foram utilizados $\mathrm{HNO}_{3} 65 \% \mathrm{v} / \mathrm{v}$ (Synth, Diadema, Brasil), bidestilado abaixo do seu ponto de ebulição, em um destilador de quartzo MA-075 (Marconi, Piracicaba, Brasil), e $\mathrm{H}_{2} \mathrm{O}_{2} 30 \%$ v/v (Merck, Darmstadt, Germany). Toda a vidraria e os materiais de laboratório foram descontaminados por imersão em $\mathrm{HNO}_{3}$ 10\% v/v por, pelo menos, $24 \mathrm{~h}$, sendo posteriormente lavados com água deionizada e secos à temperatura ambiente antes de seu uso.

\subsection{Amostras}

As amostras avaliadas foram cervejas de marcas comerciais e cervejas artesanais produzidas no Brasil. Um total de 23 amostras de cerveja dos tipos porter, lager, pale 
ale e weiss foi adquirido em mercado local (Pelotas-RS, Brasil), sendo seu conteúdo homogeneizado e desgaseificado em banho ultrassônico [40 (H) S Elmasonic, Elma, Singen, Alemanha] por 15 min antes dos experimentos ou armazenado a $-18{ }^{\circ} \mathrm{C}$. Para o desenvolvimento do método, foi utilizada uma cerveja tipo porter (amostra 1). Além desta, outras três amostras de cerveja dos tipos lager (amostra 2), pale ale (amostra 3) e weiss (amostra 4) foram utilizadas para verificar a exatidão do método, através de ensaios de adição e recuperação. Para o estudo comparativo entre as técnicas monoelementar (F AAS e F AES) e multielementar (MIP OES), novos lotes das mesmas amostras foram adquiridos, numerando-os de 5 a 8 . Após a verificação da exatidão, o método foi aplicado na determinação de $\mathrm{Ca}, \mathrm{Fe}, \mathrm{K}, \mathrm{Mg}, \mathrm{Mn}, \mathrm{Na}$, Ni e Zn em outras 15 amostras de cerveja, por MIP OES (amostras 9 a 23).

\subsection{Preparo da amostra}

O preparo das amostras foi realizado através de decomposição ácida em bloco digestor convencional (modelo MA-4025, Marconi, Brasil), utilizando-se tubos digestores acoplados a um sistema de refluxo com recirculação de água a $15^{\circ} \mathrm{C}$, proveniente de um banho termostatizado (modelo Q-214M2, Quimis, São Paulo, SP, Brasil). Em cada tubo reacional, foi introduzido um dedo frio, o que permite o uso de temperaturas superiores ao ponto de ebulição dos ácidos no bloco digestor. Embora o meio reacional não alcance a mesma temperatura aplicada no bloco digestor, outros fenômenos, como transferência de calor e turbulência, promovem uma decomposição mais rápida e eficiente.

O procedimento de digestão foi otimizado através de um planejamento estatístico e foi aplicado para todas as amostras, conforme segue: $10,0 \mathrm{~mL}$ da amostra desgaseificada e 5,0 $\mathrm{mL}$ de $\mathrm{HNO}_{3}$ foram adicionados no tubo digestor com auxílio de micropipetas, seguido de uma etapa de aquecimento com o bloco digestor a $160^{\circ} \mathrm{C}$, por 75 min. Posteriormente a um breve resfriamento, foram adicionados 2,0 $\mathrm{mL}$ de $\mathrm{H}_{2} \mathrm{O}_{2}$ e novamente aquecido por mais $18 \mathrm{~min}$, para a completa mineralização, obtendo-se, por fim, uma solução resultante que foi quantitativamente transferida e avolumada a 25,0 mL com água deionizada.

Para o desenvolvimento deste procedimento, foi empregado um delineamento composto central rotacional (DCCR), $2^{3}$ com 3 pontos centrais e 6 pontos axiais, considerando-se as seguintes variáveis independentes: volume de amostra (de 2,5 a $10 \mathrm{~mL}$ ), temperatura do bloco digestor (de 120 a $160{ }^{\circ} \mathrm{C}$ ) e tempo de decomposição (de 30 a $120 \mathrm{~min}$ ). Os ensaios foram realizados em triplicata e de forma aleatória, de acordo com a ordem das análises arranjadas pelo software Statistica 10.0 (StatSoft, Inc., Tulsa, OK, EUA), com nível de significância de 95\%; consideraram-se, como variáveis dependentes, os sinais analíticos para os elementos Zn, Mg e Na. Os volumes de amostra foram normalizados para 1,0 mL, a fim de evitar interferências dos resultados. Para verificação da exatidão do método, foram realizados ensaios de recuperação com três valores de concentração, intermediários à curva de calibração, para cada elemento investigado, os quais foram adicionados à amostra, previamente à decomposição.

Após o desenvolvimento do método, o mesmo foi aplicado a 15 amostras de cervejas artesanais (amostras 9 a 23) para a determinação de $\mathrm{Ca}, \mathrm{Fe}, \mathrm{K}, \mathrm{Mn}, \mathrm{Na}$, Ni e Zn, por MIP OES.

\subsection{Determinações por F AAS/AES e MIP OES}

As determinações monoelementares de Fe, Mg, Na e Zn foram realizadas em um espectrômetro de absorção atômica por chama Perkin Elmer AAnalyst 200 (Shelton, CT, EUA), equipado com lâmpada de deutério, para correção do sinal de fundo, e lâmpadas de cátodo oco Perkin Elmer (Shelton, CT, EUA), para cada analito. Os parâmetros instrumentais foram utilizados conforme as recomendações do fabricante. Foi utilizada a chama de ar/acetileno, com a pureza do gás oxidante de 99,7\% (Linde, São Paulo, Brasil), numa vazão de ar de 10,0 L min-1, acetileno de 2,37 L min-1 para $\mathrm{Na}$ e 2,75 L min-1 para Zn, Mg e Fe.

As determinações multielementares de $\mathrm{Ca}, \mathrm{Fe}, \mathrm{K}$, $\mathrm{Mg}, \mathrm{Mn}, \mathrm{Na}$, Ni e $\mathrm{Zn}$ foram realizadas utilizando-se um espectrômetro de emissão atômica com plasma induzido por micro-ondas MIP 4200 da Agilent Tecnologies (Melbourne, Austrália), equipado com um nebulizador e uma câmara de nebulização ciclônica de vidro dupla passagem. Nitrogênio foi extraído do ar utilizando-se um gerador de nitrogênio 4107 (Agilent Technologies, Melbourne, Austrália). O alinhamento da tocha e a calibração do comprimento de onda de cada elemento foram realizados utilizando-se uma solução de calibração em meio de $\mathrm{HNO}_{3}$ semelhante às amostras, sendo otimizados automaticamente pelo instrumento com o MP Expert Software (versão 1.5.1.0, Agilent Technologies, Melbourne, Austrália). As condições operacionais para cada elemento analisado, como comprimento de onda, posição de observação no plasma e fluxo do nebulizador, respectivamente, foram: Ca 393,366 nm, posição 10, 0,6 L min-1; Fe 371,933 nm, posição zero, 0,75 L min'-1; K 766,491 nm, posição 10, 1,0 L min-1; Mg 285,213 nm, posição 10, 0,7 L min-1; Mn 403,076 nm, posição zero, 0,9 L min-1; Na 588,995 nm, posição zero, 1,0 L min'-1; Ni 352,454 nm, posição zero, 0,7 L min-1; Zn 213,875 nm, posição zero, 0,55 L min-1.

\subsection{Parâmetros de mérito}

Os parâmetros de mérito avaliados incluem linearidade, precisão, limite de detecção (LD) e limite de quantificação (LQ). As curvas de calibração foram preparadas no mesmo meio ácido utilizado no preparo das amostras. Análise de variância (ANOVA) e teste t pareado no nível de 90\% de 
Decomposição de amostras de cerveja com sistema de refluxo para determinação monoelementar por F AAS/AES e determinação multielementar por MIP OES

Leão, P. R. P. et al.

significância foram utilizados para comparação entre os métodos utilizados.

\section{Resultados e discussões}

\subsection{Preparo de amostra}

A determinação elementar através de técnicas espectrométricas pode estar acompanhada de algumas limitações, como efeitos de matriz e particularidades dos equipamentos, o que necessita de um preparo de amostra eficiente, previamente às determinações. Um exemplo disso é o MIP OES, que usa um plasma de nitrogênio, menor se comparado ao plasma induzido de argônio. Esse plasma de menor energia reflete em limitações ao teor de acidez (máximo de $5 \%$ v/v) e sólido dissolvido ( $3 \% \mathrm{~m} / \mathrm{v})$, de acordo com o fabricante do equipamento. Assim, a etapa de preparo das amostras é fundamental para a eficiência das determinações, para diminuir interferências no equipamento, como depósitos na tocha, não permitindo, por exemplo, o uso de amostragem direta ou a matriz na forma de uma suspensão. O mesmo acontece com o F AAS/AES, que, ao ser utilizado com amostras sem prévia decomposição, causa incrustação de partículas no nebulizador ou queimador. Em amostras de cerveja, embora sejam amostras na forma líquida, nem mesmo um processo de centrifugação elimina esse problema, relatado por diferentes autores, originando sinais de absorção/emissão flutuantes e não reprodutíveis, além de oscilações na chama (BELLIDO-MILLA et al., 2000; HERGENREDER, 1991; MATUSIEWICZ; KOPRAS, 1997; BORRIELLO; SCIAUDONE, 1980).
Os resultados obtidos a partir do DCCR para $\mathrm{Na}$, Mg e Zn, pelas técnicas de F AAS/AES, estão apresentados na Tabela 1, os quais permitem avaliar os parâmetros estatisticamente significativos no preparo da amostra, utilizando-se decomposição ácida com o sistema de refluxo. O Diagrama de Pareto, para cada analito, é apresentado na Figura 1. Entre todas as variáveis investigadas e de acordo com o Diagrama de Pareto, para Zn (Figura 1A), apenas a temperatura foi estatisticamente significativa; para Mg (Figura 1B), apenas o volume da amostra, e para Na (Figura 1C), nenhuma das variáveis se mostrou significativa. Os modelos gerados estão adequados para a descrição dos resultados e as superfícies de respostas são apresentadas na Figura 2.

Alguns autores relatam o uso de maiores volumes de amostra, variando de 10 a $50 \mathrm{~mL}$ na abertura das amostras, para determinação dos minerais e contaminantes em cerveja (POHL; PRUSISZ, 2007; BELLIDO-MILLA et al., 2000; ALCÁZAR et al., 2002). Neste estudo, para Zn (Figura 2A), os valores de volume estudados não apresentaram diferença estatisticamente significativa, enquanto que, para Mg (Figura 2B), os valores inferiores ao ponto central foram os que originaram resultados satisfatórios. Entretanto, quando volumes abaixo do ponto central foram utilizados, não era possível realizar a determinação de elementos em baixa concentração. Ao utilizar o maior volume (10 mL), foi possível realizar a determinação dos elementos minoritários nas amostras, bem como o Mg, que se apresenta em maior concentração. Portanto, foi escolhido o maior volume de amostra estudado (10 mL), pois este mostrou ser mais eficiente para todos os analitos, com melhores LDs e LQs.

Tabela 1. Matriz do DCCR para a decomposição da amostra e determinações de Mg, Na e Zn por F AAS

\begin{tabular}{|c|c|c|c|c|c|c|}
\hline \multirow[b]{2}{*}{ Ensaios } & \multicolumn{3}{|c|}{ Variáveis independentes } & \multicolumn{3}{|c|}{ Variáveis dependentes } \\
\hline & $\begin{array}{c}\text { Volume de } \\
\text { amostra (mL) }\end{array}$ & $\begin{array}{c}\text { Temperatura } \\
\left({ }^{\circ} \mathbf{C}\right)\end{array}$ & $\begin{array}{c}\text { Tempo } \\
\text { (min) }\end{array}$ & Mg & $\mathrm{Na}$ & $\mathrm{Zn}$ \\
\hline 1 & 4,4 & 130 & 48 & 0,4180 & 5729 & 0,8567 \\
\hline 2 & 8,2 & 130 & 48 & 0,2361 & 3941 & 0,8390 \\
\hline 3 & 4,4 & 150 & 48 & 0,4136 & 5781 & 0,8347 \\
\hline 4 & 8,2 & 150 & 48 & 0,2341 & 3885 & 0,8310 \\
\hline 5 & 4,4 & 130 & 130 & 0,4179 & 5636 & 0,8143 \\
\hline 6 & 8,2 & 130 & 130 & 0,2335 & 3788 & 1,2590 \\
\hline 7 & 4,4 & 150 & 130 & 0,4120 & 5749 & 0,8143 \\
\hline 8 & 8,2 & 150 & 130 & 0,2352 & 3962 & 1,2580 \\
\hline 9 & 2,5 & 130 & 75 & 0,3905 & 4839 & 0,8067 \\
\hline 10 & 10,0 & 130 & 75 & 0,1951 & 3471 & 0,8807 \\
\hline 11 & 6,30 & 120 & 75 & 0,7529 & 11640 & 1,2757 \\
\hline 12 & 6,30 & 160 & 75 & 0,2956 & 4491 & 1,2653 \\
\hline 13 & 6,30 & 140 & 30 & 0,2973 & 4459 & 0,8067 \\
\hline 14 & 6,30 & 140 & 120 & 0,2952 & 4477 & 0,8790 \\
\hline $15(\mathrm{C})$ & 6,30 & 140 & 75 & 0,2985 & 4587 & 0,8623 \\
\hline $16(\mathrm{C})$ & 6,30 & 140 & 75 & 0,2989 & 4655 & 0,8617 \\
\hline $17(\mathrm{C})$ & 6,30 & 140 & 75 & 0,2993 & 4620 & 0,8683 \\
\hline
\end{tabular}


Decomposição de amostras de cerveja com sistema de refluxo para determinação monoelementar por F AAS/AES e determinação multielementar por MIP OES

Leão, P. R. P. et al.

A

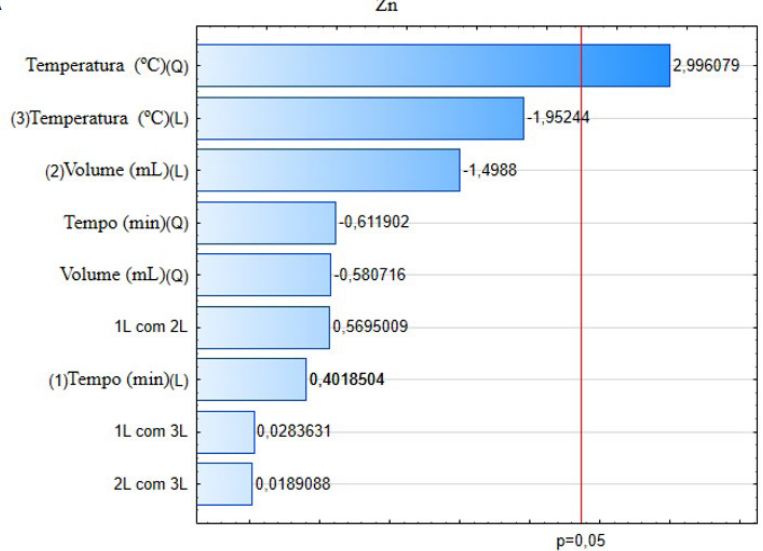

B

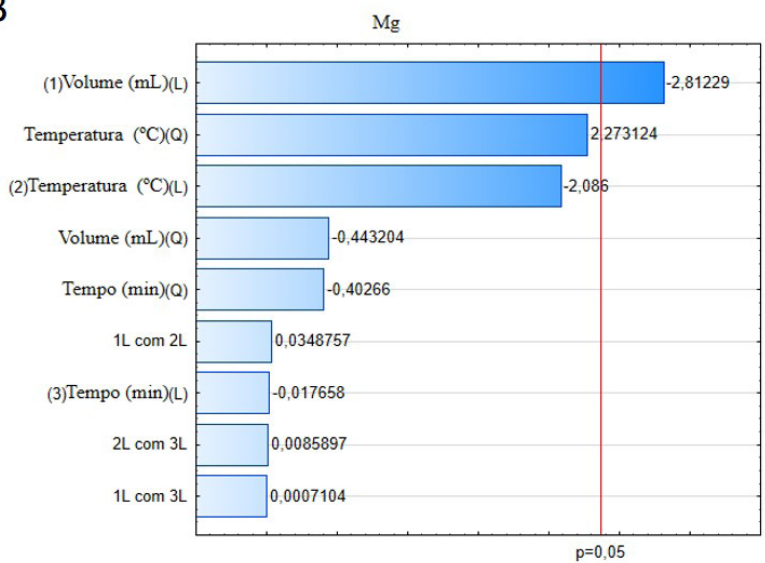

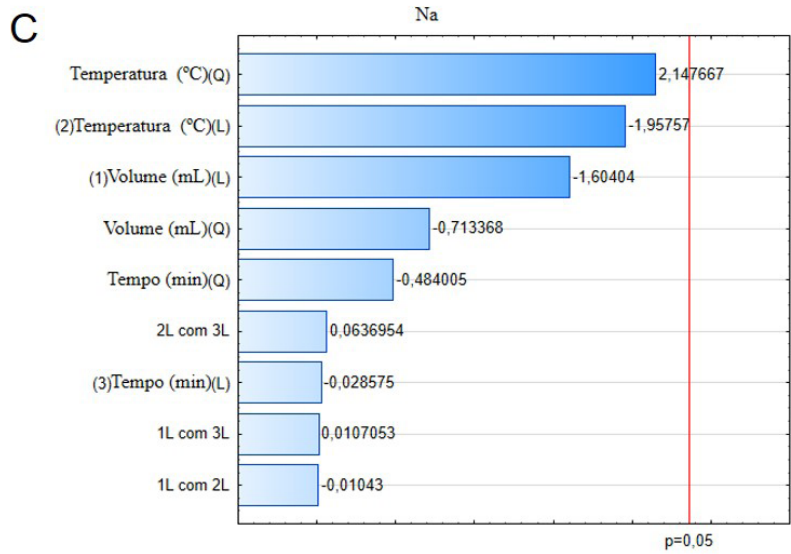

Figura 1. Diagrama de Pareto a $95 \%$ de confiança para Zn (A), Mg (B) e Na (C).

O uso da temperatura um pouco acima do ponto de ebulição do ácido nítrico para decomposição da amostra é relatado na literatura, em muitos estudos (LOPEZ et al., 1998; MENA et al., 1996; HUSÁKOVÁ et al., 2007). Na Figura 2A, observa-se que, para o Zn, as temperaturas abaixo de $130{ }^{\circ} \mathrm{C}$ e acima de $150^{\circ} \mathrm{C}$ geram resultados satisfatórios. Assim, foi escolhido o maior valor de temperatura $\left(160^{\circ} \mathrm{C}\right)$, para permitir, com melhor eficiência, o rompimento de ligações entre os elementos e peptídeos na cerveja, que atuam na formação da espuma (PALMER, 2006). Além dos benefícios já comentados anteriormente, como uma melhor eficiência na transferência de calor e aumento na turbulência do meio reacional, maior valor de temperatura torna o processo mais eficiente, em um tempo menor para o preparo das amostras de cerveja.

Para períodos mais longos de decomposição, foi observada a formação de material em suspensão, o que pode causar entupimento do sistema de nebulização dos equipamentos. Para evitar este problema durante as medições instrumentais, foi escolhido um tempo intermediário (93 minutos).

Durante a decomposição da amostra, foi utilizado o volume de $5 \mathrm{~mL}$ de $\mathrm{HNO}_{3}$ o qual, em testes preliminares, mostrou-se necessário para a completa decomposição da matriz, para não ocorrerem depósitos na tocha e inviabilizar as determinações. Por tratar-se de uma amostra líquida em meio aquoso, altas diluições do ácido poderiam eliminar o seu poder oxidante.

Após a análise do DCCR, as seguintes condições de decomposição da amostra, para determinação multielementar em cerveja, foram aplicadas: $10,0 \mathrm{~mL}$ de amostra, $5,0 \mathrm{~mL}$ de $\mathrm{HNO}_{3}, 160^{\circ} \mathrm{C}$ para a temperatura do bloco digestor e 93 min de aquecimento, sendo que $18 \mathrm{~min}$ desse tempo foram aplicados após a adição de 2,0 mL de $\mathrm{H}_{2} \mathrm{O}_{2}$. A precisão e a exatidão do método foram avaliadas pelos ensaios de recuperação. Além da cerveja porter (amostra 3), utilizada no desenvolvimento do método, os ensaios de recuperação foram realizados também em cerveja pale ale, lager e weiss, cujos resultados estão apresentados nas Tabelas 2 e 3. Para isso, diferentes técnicas analíticas foram utilizadas: F AES para $\mathrm{Na}$, F AAS para Fe, Mg e Zn e MIP OES para Ca, K, Mn e Ni. Os valores obtidos pelo teste de recuperação variaram entre 80 e 120\%, para todos os analitos, apresentando uma média de recuperação de 101, 99 e 101, para FAAS, F AES e MIP OES, respectivamente. Esses dados mostram que o método de preparo de amostra pode ser aplicado com segurança nas diferentes técnicas de espectrometria 


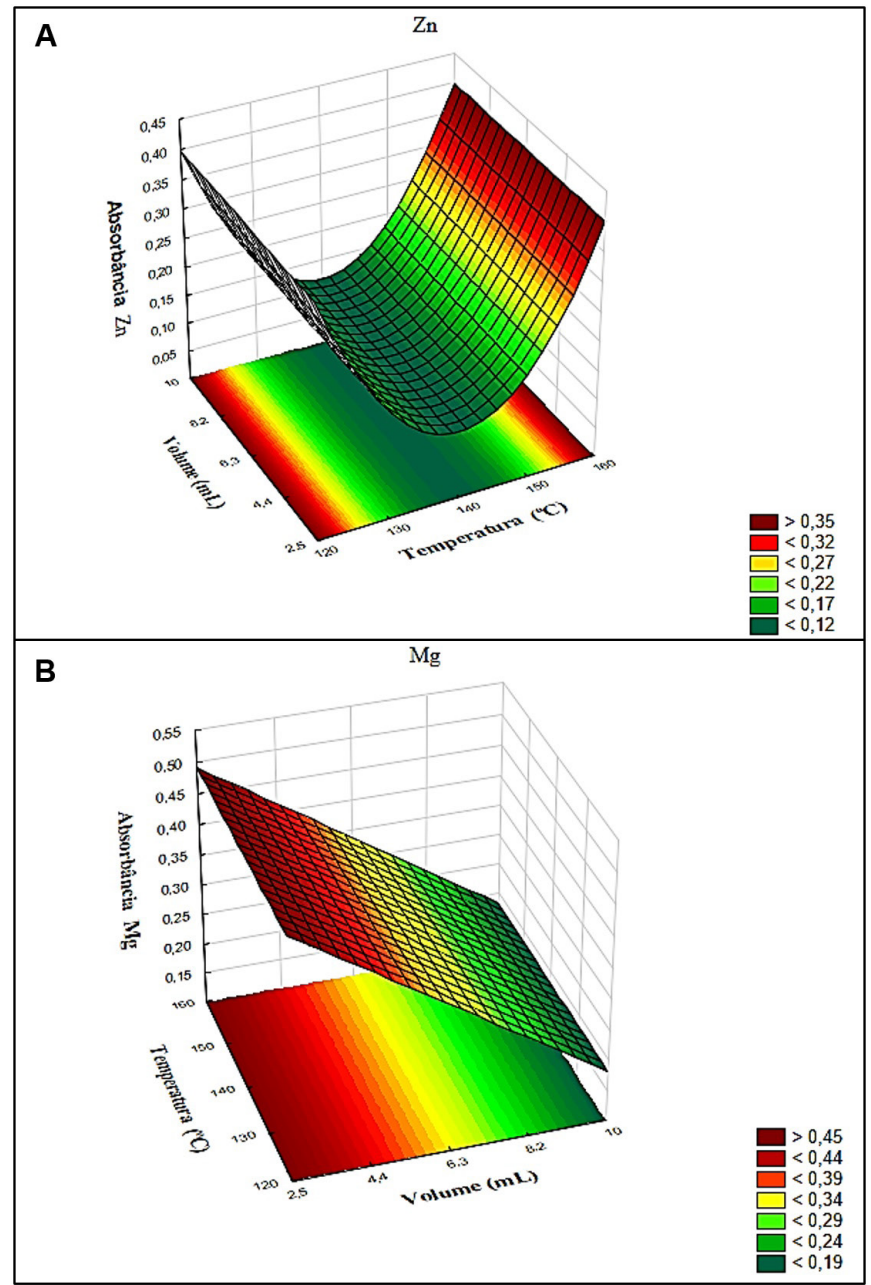

Figura 2. Gráficos de superfície de respostas: $(A)$ relação entre volume $(\mathrm{mL}) \times$ temperatura $\left({ }^{\circ} \mathrm{C}\right)$ para $\mathrm{Zn}$; (B) relação entre temperatura $\left({ }^{\circ} \mathrm{C}\right) \times$ volume $(\mathrm{mL})$ para $\mathrm{Mg}$.

atômica, pois este forneceu resultados exatos e precisos, com um RSD médio de 3,2\%.

No estudo comparativo entre as determinações mono e multielementares, para a determinação de $\mathrm{Na}$, Fe, Mg e Zn (Tabela 4), o teste T pareado, considerando-se nível de confiança de 90\%, mostrou que não há diferenças significativas entre as técnicas utilizadas na determinação dos analitos.

\subsection{Resultados analíticos}

As figuras de mérito para $\mathrm{Ca}, \mathrm{Fe}, \mathrm{K}, \mathrm{Mg}, \mathrm{Mn}$, $\mathrm{Na}$, Ni e Zn são apresentadas na Tabela 5. Os limites de quantificação (LQ) e detecção (LD) obtidos para o método proposto ficaram abaixo dos valores estipulados pela ANVISA, para determinação de Zn e Ni, que são de 1 e $0,1 \mathrm{mg} \mathrm{L}^{-1}$, respectivamente (BRASIL, 1965). Para os demais analitos estudados, não existem limites máximos na legislação brasileira. Além disso, observa-se que a determinação dos elementos por MIP OES é mais sensível frente à técnica monoelementar, apresentando melhores coeficientes angulares das curvas de calibração (aumento de sensibilidade), o qual, aliado a uma melhora na precisão das determinações (menor desvio padrão), forneceu baixos valores de LDs e LQs. Entretanto, no F AAS para o Mg e Zn, os valores de LD's e LQ's apresentaram-se menores do que no MIP OES, evidenciando também sua boa sensibilidade. Os valores de $\mathrm{R}^{2}$ foram de 0,999 para quase todos os analitos, com exceção do Fe, que foi de 0,996 .

A Tabela 6 apresenta os resultados da aplicação do método proposto na determinação multielementar de $\mathrm{Ca}, \mathrm{Fe}, \mathrm{K}, \mathrm{Mg}, \mathrm{Mn}$, Na, Ni e Zn (mg L-1) por MIP OES, nas amostras de cervejas de diferentes tipos e fabricantes. A identificação das amostras é apresentada de maneira numérica e aleatória, em razão da disponibilidade de obtenção no mercado, já que grande parte das amostras são cervejas especiais; assim, conforme a obtenção e a chegada ao laboratório, as amostras foram recebendo a numeração sequencial para realização das determinações. Os valores encontrados assemelham-se aos da literatura (MATSUSHIGE; OLIVEIRA, 1993; LEWIS; BAMFORTH, 2006). O potássio foi o elemento estudado que apresentou os maiores teores em todas as amostras. Sua origem na cerveja, assim como outros íons, é proveniente do malte, o que está de acordo com outros estudos realizados, influenciando na qualidade final da bebida (LEWIS; BAMFORTH, 2006; BELLIDO-MILLA et al., 2000; ALCÁZAR et al., 2002; MARCANO et al., 2010). Para alguns elementos, como Mn, Mg e K, a cerveja pilsen apresentou os menores valores. Possivelmente, por ser uma cerveja de menor turbidez e sabores mais suaves, pode levar em sua formulação menor quantidade de matéria-prima, como o lúpulo e o malte (KEUKELEIRE, 2000), o que acarreta menores teores de alguns elementos. As cervejas dos estilos weiss (de trigo) e escuras são cervejas de maior turbidez e coloração mais intensa do que as de outros estilos (MOURA-NUNES et al., 2016). Como a etapa de clarificação remove componentes da cerveja, é esperado que as amostras com maior turbidez apresentassem maiores teores de metais que as demais cervejas, o que não aconteceu.

O níquel foi um elemento encontrado em todas as cervejas, em um teor médio de 0,1 $\mathrm{mg} \mathrm{L}^{-1}$. Por ser um micronutriente essencial para as plantas (WYRZYKOWSKA et al., 2001), sua origem na cerveja pode estar relacionada ao lúpulo ou à cevada. O Zn apresenta baixos teores na cerveja (em torno de $1 \mathrm{mg} \mathrm{L}^{-1}$ ) e embora seja um elemento importante para a fermentação pelas leveduras, altos teores poderiam influenciar negativamente na formação da espuma, assim como outros elementos, como o Fe (POHL, 2008; PALMER, 2006). O Fe, Mn e Ni estão diretamente relacionados à estabilidade oxidativa da cerveja, atuando como pró-oxidantes e na formação de 
Decomposição de amostras de cerveja com sistema de refluxo para determinação monoelementar por F AAS/AES e determinação multielementar por MIP OES

Leão, P. R. P. et al.
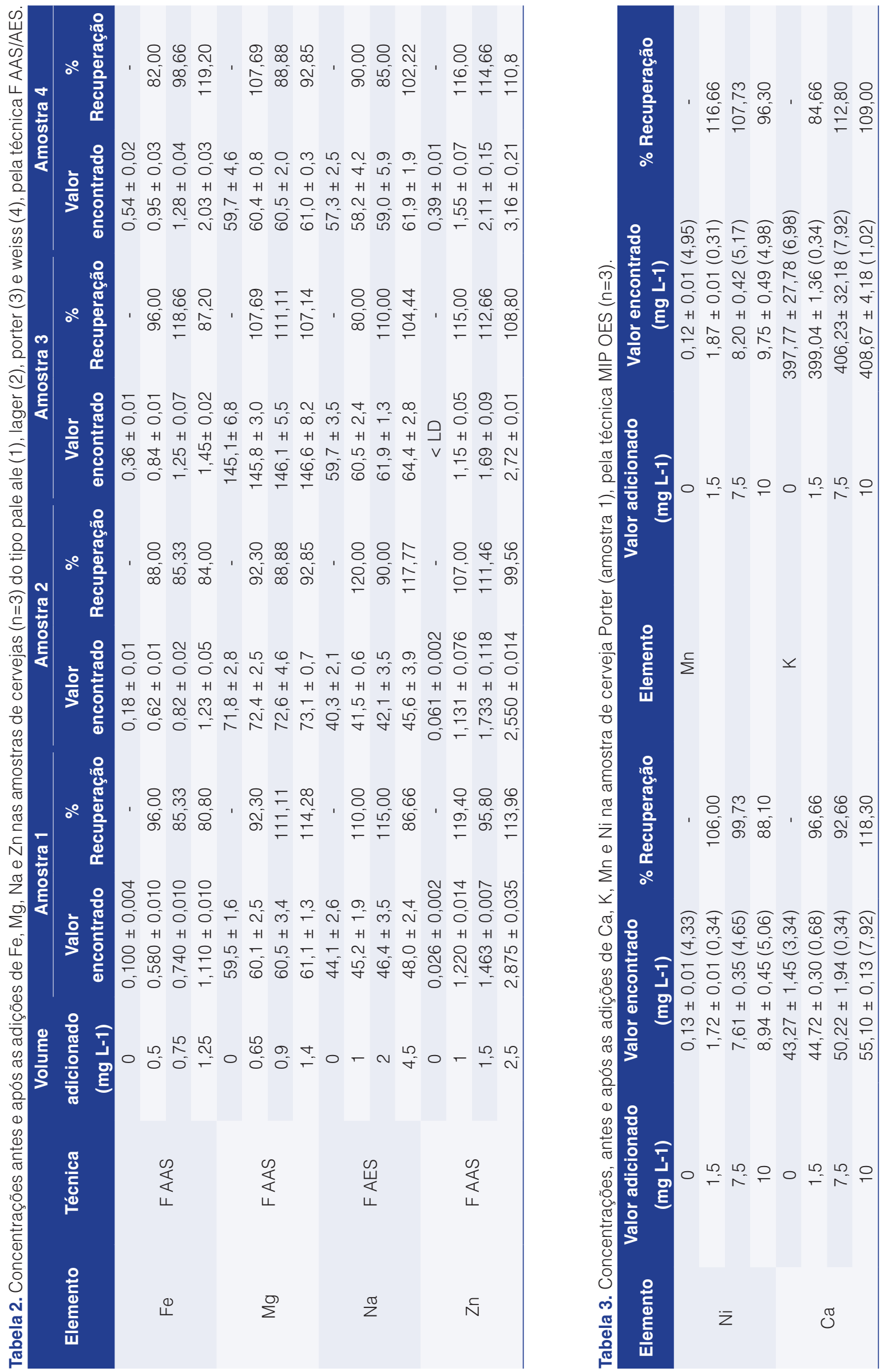
Decomposição de amostras de cerveja com sistema de refluxo para determinação monoelementar por $F$ AAS/AES e determinação multielementar por MIP OES

Leão, P. R. P. et al.

Tabela 4. Concentrações de Na, Mg, Fe e Zn nas amostras de cerveja (porter, lager, pale ale e weiss), por F AAS/F AES e MIP OES $(n=3)$.

\begin{tabular}{|c|c|c|c|c|}
\hline \multirow{3}{*}{ Amostra } & \multicolumn{4}{|c|}{$\dot{\mathrm{X}} \pm \mathrm{DP}\left(\mathrm{mg} \mathrm{L^{-1 }}\right)$} \\
\hline & \multicolumn{2}{|c|}{$\mathrm{Fe}$} & \multicolumn{2}{|c|}{ Mg } \\
\hline & F AAS & MIP OES & F AAS & MIP OES \\
\hline 5 & $0,273 \pm 0,003$ & $0,275 \pm 0,001$ & $89,47 \pm 3,35$ & $90,52 \pm 0,85$ \\
\hline 6 & $0,113 \pm 0,004$ & $0,100 \pm 0,001$ & $63,20 \pm 0,69$ & $61,51 \pm 0,35$ \\
\hline 7 & $0,177 \pm 0,004$ & $0,173 \pm 0,006$ & $78,75 \pm 2,14$ & $77,47 \pm 1,13$ \\
\hline 8 & $0,111 \pm 0,008$ & $0,100 \pm 0,001$ & $82,53 \pm 1,10$ & $83,02 \pm 0,04$ \\
\hline \multirow{2}{*}{ Amostra } & \multicolumn{2}{|c|}{$\mathrm{Na}$} & \multicolumn{2}{|c|}{$\mathbf{Z n}$} \\
\hline & F AES & MIP OES & F AAS & MIP OES \\
\hline 5 & $41,80 \pm 0,92$ & $42,07 \pm 0,15$ & $1,200 \pm 0,017$ & $1,143 \pm 0,012$ \\
\hline 6 & $25,55 \pm 0,61$ & $26,79 \pm 0,52$ & $0,994 \pm 0,022$ & $1,030 \pm 0,010$ \\
\hline 7 & $61,25 \pm 0,56$ & $58,52 \pm 0,97$ & $0,764 \pm 0,009$ & $0,760 \pm 0,010$ \\
\hline 8 & $38,17 \pm 1,23$ & $39,27 \pm 0,63$ & $0,857 \pm 0,008$ & $0,867 \pm 0,014$ \\
\hline
\end{tabular}

Amostra 5: cerveja porter; Amostra 6: cerveja lager; Amostra 7: cerveja pale ale; Amostra 8: cerveja weiss.

Tabela 5. Figuras de mérito para a determinação de $\mathrm{Ca}$, Fe, $\mathrm{K}, \mathrm{Mg}, \mathrm{Mn}$, Na, $\mathrm{Ni}$ e $\mathrm{Zn}$ em amostras de cerveja, por $\mathrm{F}$ AES, F AAS e MIP OES.

\begin{tabular}{|c|c|c|c|c|c|c|c|c|}
\hline \multirow[b]{2}{*}{ Elemento } & \multirow[b]{2}{*}{ Técnica } & \multirow[b]{2}{*}{$\begin{array}{c}a \\
\left(L \mathrm{mg}^{-1}\right)\end{array}$} & \multirow[b]{2}{*}{$\mathbf{R}^{2}$} & \multirow[b]{2}{*}{$\begin{array}{c}\text { Faixa linear } \\
\left(\mathrm{mg} \mathrm{L}^{-1}\right)\end{array}$} & \multicolumn{2}{|c|}{ Instrumental } & \multicolumn{2}{|c|}{ Método } \\
\hline & & & & & $\begin{array}{c}\text { LD } \\
\left(\mu g L^{-1}\right)\end{array}$ & $\begin{array}{c}\mathrm{LQ} \\
\left(\mu \mathrm{g} \mathrm{L}^{-1}\right)\end{array}$ & $\begin{array}{c}\text { LD } \\
\left(\mu g L^{-1}\right)\end{array}$ & $\begin{array}{c}\mathrm{LQ} \\
\left(\mu \mathrm{L} \mathrm{L}^{-1}\right)\end{array}$ \\
\hline $\mathrm{Ca}$ & MIP OES & 605804 & 0,999 & $0,5-5,0$ & 3,0 & 8,0 & 7,0 & 20,0 \\
\hline \multirow{2}{*}{$\mathrm{Fe}$} & F AAS & 0,0827 & 0,995 & $0,15-0,75$ & 10,0 & 40,0 & 25,0 & 100,0 \\
\hline & MIP OES & 14886 & 0,996 & & 5,0 & 20,0 & 10,0 & 50,0 \\
\hline K & MIP OES & 123229 & 0,999 & $0,5-5,0$ & 1,0 & 2,0 & 2,0 & 5,0 \\
\hline \multirow{2}{*}{$\mathrm{Mg}$} & F AAS & 1,1017 & 0,998 & $0,2-0,6$ & 2,0 & 5,0 & 5,0 & 12,0 \\
\hline & MIP OES & 321698 & 0,999 & & 3,0 & 8,0 & 7,0 & 20,0 \\
\hline $\mathrm{Mn}$ & MIP OES & 58827 & 0,999 & $0,5-5,0$ & 0,4 & 1,0 & 1,0 & 3,0 \\
\hline \multirow{2}{*}{$\mathrm{Na}$} & F AES & 9445 & 0,995 & $0,1-2,0$ & 1,0 & 3,0 & 3,0 & 7,0 \\
\hline & MIP OES & 630543 & 0,999 & & 1,0 & 2,0 & 2,0 & 5,0 \\
\hline $\mathrm{Ni}$ & MIP OES & 29508 & 0,999 & $0,5-5,0$ & 0,4 & 1,0 & 1,0 & 2,5 \\
\hline \multirow{2}{*}{$\mathrm{Zn}$} & F AAS & 0,4566 & 0,991 & $0,10-2,0$ & 3,0 & 8,0 & 7,0 & 20,0 \\
\hline & MIP OES & 20486 & 0,999 & & 6,0 & 15,0 & 15,0 & 40,0 \\
\hline
\end{tabular}

a: coeficiente angular da curva de calibração; R²: coeficiente de correlação linear; LD: limite de detecção; LQ: limite de quantificação.

Tabela 6. Concentrações de Ca, Fe, K, Mg, Mn, Na, Ni e Zn em 15 amostras de cerveja, por MIP OES ( $\mathrm{n}=3)$.

\begin{tabular}{cccccccccc}
\multicolumn{2}{c}{ Amostra } & $\mathbf{C a}$ & $\mathbf{F e}$ & $\mathbf{K}$ & $\mathbf{M g}$ & $\mathbf{M n}$ & $\mathbf{N a}$ & $\mathbf{N i}$ & $\mathbf{Z n}$ \\
Pale Ale & 11 & $9,57 \pm 0,02$ & $0,213 \pm 0,006$ & $445,2 \pm 11,4$ & $75,33 \pm 1,71$ & $0,117 \pm 0,006$ & $45,47 \pm 2,03$ & $0,077 \pm 0,006$ & $1,060 \pm 0,057$ \\
& 21 & $27,25 \pm 0,03$ & $0,257 \pm 0,012$ & $588,2 \pm 1,5$ & $70,70 \pm 0,39$ & $0,133 \pm 0,006$ & $33,02 \pm 1,48$ & $0,060 \pm 0,001$ & $0,733 \pm 0,021$ \\
& 23 & $20,93 \pm 0,23$ & $0,113 \pm 0,006$ & $636,0 \pm 7,3$ & $69,34 \pm 1,75$ & $0,263 \pm 0,006$ & $91,61 \pm 3,55$ & $0,070 \pm 0,001$ & $0,675 \pm 0,007$ \\
Lager & 10 & $20,59 \pm 0,42$ & $0,170 \pm 0,001$ & $437,3 \pm 4,3$ & $59,39 \pm 2,75$ & $0,130 \pm 0,001$ & $27,85 \pm 1,61$ & $0,067 \pm 0,006$ & $1,030 \pm 0,001$ \\
& 15 & $127,00 \pm 2,96$ & $0,065 \pm 0,007$ & $359,9 \pm 5,0$ & $96,59 \pm 1,39$ & $0,247 \pm 0,012$ & $54,20 \pm 0,99$ & $0,073 \pm 0,006$ & $0,550 \pm 0,001$ \\
& 17 & $47,59 \pm 0,21$ & $0,080 \pm 0,001$ & $446,1 \pm 1,6$ & $80,05 \pm 0,76$ & $0,073 \pm 0,006$ & $36,27 \pm 0,21$ & $0,080 \pm 0,001$ & $1,280 \pm 0,028$ \\
\multirow{5}{*}{ Bock } & 18 & $42,27 \pm 1,12$ & $0,075 \pm 0,007$ & $452,9 \pm 5,2$ & $69,56 \pm 0,17$ & $0,095 \pm 0,007$ & $72,92 \pm 0,52$ & $0,160 \pm 0,014$ & $2,335 \pm 0,163$ \\
& 19 & $27,39 \pm 0,25$ & $0,080 \pm 0,001$ & $567,2 \pm 7,0$ & $91,30 \pm 0,66$ & $0,110 \pm 0,001$ & $41,72 \pm 0,32$ & $0,095 \pm 0,007$ & $1,143 \pm 0,012$ \\
& 20 & $43,51 \pm 1,34$ & $<0,050$ & $248,2 \pm 0,4$ & $63,05 \pm 0,12$ & $0,095 \pm 0,007$ & $34,84 \pm 0,20$ & $0,165 \pm 0,007$ & $1,670 \pm 0,001$ \\
Weiss & 9 & $20,89 \pm 0,08$ & $0,185 \pm 0,007$ & $284,1 \pm 4,7$ & $63,28 \pm 1,88$ & $0,240 \pm 0,010$ & $60,82 \pm 3,42$ & $0,093 \pm 0,006$ & $0,760 \pm 0,014$ \\
& 16 & $10,22 \pm 0,09$ & $<0,050$ & $499,7 \pm 17,8$ & $79,95 \pm 2,82$ & $0,217 \pm 0,006$ & $39,57 \pm 1,32$ & $0,093 \pm 0,006$ & $1,243 \pm 0,031$ \\
& 22 & $5,68 \pm 0,14$ & $0,145 \pm 0,007$ & $455,3 \pm 19,2$ & $91,76 \pm 3,51$ & $0,370 \pm 0,010$ & $61,23 \pm 1,90$ & $0,070 \pm 0,001$ & $0,960 \pm 0,028$ \\
Pilsen & 12 & $35,60 \pm 0,29$ & $<0,050$ & $245,6 \pm 3,4$ & $45,65 \pm 1,06$ & $0,060 \pm 0,001$ & $68,01 \pm 2,06$ & $0,067 \pm 0,006$ & $1,825 \pm 0,205$ \\
& 13 & $74,86 \pm 0,51$ & $0,125 \pm 0,007$ & $245,9 \pm 2,9$ & $49,88 \pm 0,74$ & $0,077 \pm 0,006$ & $59,95 \pm 3,10$ & $0,075 \pm 0,007$ & $2,580 \pm 0,325$ \\
& 14 & $50,34 \pm 1,96$ & $<0,050$ & $253,6 \pm 5,5$ & $55,69 \pm 1,93$ & $0,080 \pm 0,001$ & $58,58 \pm 1,81$ & $0,073 \pm 0,006$ & $1,980 \pm 0,099$ \\
\hline
\end{tabular}


flavours indesejáveis, o que evidencia sua importância no monitoramento e na escolha da matéria-prima, já que os cereais utilizados na produção são fonte desses elementos para a cerveja (ZUFALL; TYRELL, 2008). De acordo com Krüger e Anger (1990), os valores encontrados para Fe e $\mathrm{Mn}$, nas amostras, estão abaixo de valores representativos para desencadear reações oxidativas prejudiciais às características sensoriais da cerveja. As diferenças encontradas nos valores dos analitos, neste estudo, podem estar relacionadas a diferentes características das cervejas, como flavour, espuma e formação de aroma entre os diferentes estilos ou até mesmo entre diferentes marcas com o mesmo estilo.

Das amostras avaliadas, apenas duas cervejas apresentaram concentração de Ni acima do limite estabelecido pela legislação brasileira (> 0,1 $\left.\mathrm{mg} \mathrm{L}^{-1}\right)$, e 10 amostras, acima do limite para Zn (> $1 \mathrm{mg} \mathrm{L}^{-1}$ ) (35); dentre estas, por exemplo, as amostras 18 e 20, ambas cervejas escuras, apresentaram elevados teores de Ni e Zn. Verifica-se mais uma vez a importância de um método preciso e com baixos limites de detecção e quantificação para determinação desses elementos, garantindo a qualidade e a segurança dos produtos destinados ao consumo humano.

ANOVA e teste t pareado foram utilizados para determinar diferenças estatisticamente significativas entre médias das amostras. Entre a maioria das amostras de cervejas, houve diferenças estatísticas a um $p<0,05$, na maioria dos elementos. Isso pode ocorrer devido a diferentes matérias-primas empregadas na fabricação, assim como a sua origem. Outro fator que contribui para essa diferença entre os valores das cervejas é a armazenagem passível de contaminações. De maneira geral, não existe uma padronização na fabricação das cervejas artesanais, assim como ocorre nas grandes indústrias. Assim, se fazem necessárias determinações constantes para evitar variações dos minerais e evitar possíveis contaminações.

\section{Conclusão}

Este trabalho mostrou a viabilidade do método proposto de decomposição ácida em sistema de refluxo, para amostras de cerveja. O auxílio do planejamento estatístico propiciou a obtenção de resultados satisfatórios, independentemente da técnica utilizada nas determinações. O método desenvolvido mostrou ser de fácil aplicação e de baixo custo, quando comparado com métodos já existentes. Além disso, as amostras decompostas puderam ser avaliadas em diferentes equipamentos, evidenciando a eficiência e a versatilidade do método de preparo das amostras. O uso do MIP OES possibilitou uma maior frequência analítica, pois permite uma análise multielementar, com menores $L Q$, reduzindo significativamente os custos das determinações, já que necessita de pequena quantidade de amostra e não requer o uso de gases especiais.
Entre as amostras avaliadas, não há diferenças significativas entre os estilos de cerveja, em relação ao teor dos elementos estudados. Portanto, fica evidente a importância de um método simples e efetivo de monitoramento de metais nas cervejas, já que os mesmos exercem importante papel nas características do produto, bem como no controle de qualidade, evitando contaminação e padronizando a produção.

\section{Agradecimentos}

Os autores agradecem ao Conselho Nacional de Desenvolvimento Científico e Tecnológico (CNPq, Processo $n^{\circ}$ 447373/2014-5) e à Coordenação de Aperfeiçoamento de Pessoal de Nível Superior (CAPES, AUXPE 2973/2011) pelo apoio financeiro e bolsas de estudo.

\section{Referências}

AGU, R. C. Comparative study of experimental beers brewed from millet, sorghum and barley malts. Process Biochemistry, v. 30 , n. 4 , p. 311-315, 1995. http://dx.doi.org/10.1016/00329592(95)87039-3.

ALCÁZAR, A.; PABlos, F.; MARTín, M. A.; GOnZÁleZ, A. G. Multivariate characterisation of beers according to their mineral content. Talanta, v. 57, n. 1, p. 45-52, 2002. PMid:18968603. http://dx.doi.org/10.1016/S0039-9140(01)00670-1.

ASSOCIAÇÃO BRASILEIRA DA INDÚSTRIA DA CERVEJA CERVBRASIL. Anuário 2016. São Paulo: CERVBRASIL, 2016. Disponível em: <http://www.cervbrasil.org.br/paginas/index. php>. Acesso em: 20 abr. 2017.

BAMFORTH, C. W. Nutritional aspects of beer: a review. Nutrition Research, v. 22, n. 1-2, p. 227-237, 2002. http://dx.doi.org/10.1016/ S0271-5317(01)00360-8.

BELLIDO-MILLA, D.; MORENO-PEREZ, J. M.; HERNÁNDEZARTIGA, M. Differentiation and classification of beers with flame atomic spectrometry and molecular absorption spectrometry and sample preparation assisted by microwaves. Spectrochimica Acta Part B: Atomic Spectroscopy, v. 55, n. 7, p. 855-864, 2000. http://dx.doi.org/10.1016/S0584-8547(00)00164-6.

BORRIELLO, R.; SCIAUDONE, G. Zinc, copper, iron and lead in bottled and canned beer by atomic absorption spectroscopy. Atomic Spectroscopy, v. 1, p. 131-132, 1980.

BRASIL. Agência Nacional de Vigilância Sanitária - ANVISA. Decreto $n^{\circ} 55.871$, de 26 de março de 1965. Diário Oficial [da] República Federativa do Brasil, Brasília, DF, 9 abr. 1965. Disponível em: <http://portal.anvisa.gov.br/>. Acesso em: 20 nov. 2015.

CNBC. Top 20 beer drinking countries. 2017. Disponível em: <http://www.cnbc.com/2008/09/22/Top-20-Beer-Drinking-Countries. html?slide=19>. Acesso em: 28 jun. 2017. 
Decomposição de amostras de cerveja com sistema de refluxo para determinação monoelementar por F AAS/AES e determinação multielementar por MIP OES

Leão, P. R. P. et al.

FERREIRA, S. L. C.; JESUS, R. M.; MATOS, G. D.; ANDRADE, J. B.; BRUNS, R. E.; SANTOS, W. N. L.; CAVALCANTE, D. D.; VALE, M. G. R.; DESSUY, M. B. Multivariate optimization and validation of an analytical method for the determination of cadmium in wines employing ET AAS. Journal of the Brazilian Chemical Society, v. 20, n. 4, p. 788-794, 2009. http://dx.doi.org/10.1590/ S0103-50532009000400022.

HERGENREDER, R. L. Determination of iron in beer by flow injection-flame atomic absorption spectroscopy. Atomic Spectroscopy, v. 12, p. 74-76, 1991.

HUSÁKOVÁ, L.; ČERNOHORSKÝ, T.; ŠRÁMKOVÁ, J.; VAVRUŠOVÁ, $\mathrm{L}$. Direct determination of arsenic in beer by electrothermal atomic absorption spectrometry with deuterium background correction (D2-ET-AAS). Food Chemistry, v. 105, n. 1, p. 286-292, 2007. http://dx.doi.org/10.1016/j.foodchem.2006.11.025.

KEUKELEIRE, D. Fundamentals of beer and hop chemistry. Química Nova, v. 23, n. 1, p. 108-112, 2000. http://dx.doi. org/10.1590/S0100-40422000000100019.

KRÜGER, E.; ANGER, H. M. Kennzahlen zur betriebskontrolle und qualitätsbeschreibung in der brauwirtschaft. Hamburg: B. Behr's, 1990.

LEWIS, M. J.; BAMFORTH, C. W. Essays in brewing science. California: Springer Science Business Media, 2006.

LI, Y.; VAN LOON, J. C.; BAREFOOT, R. R. Preconcentration of trace elements in potable liquids by means of a liquid membrane emulsion for flame atomic absorption determination. Fresenius' Journal of Analytical Chemistry, v. 345, n. 6, p. 467-470, 1993. http://dx.doi.org/10.1007/BF00325628.

LOPEZ, F. F.; CABRERA, C.; LORENZO, M. L.; LOPEZ, M. C. Aluminium levels in wine, beer and other alcoholic beverages consumed in Spain. The Science of the Total Environment, $v$. 220, n. 1, p. 1-9, 1998. PMid:9800382. http://dx.doi.org/10.1016/ S0048-9697(98)00214-9

MARCANO, E.; GÓMEZ, C.; BENZO, Z.; LAINE, J. Estudio preliminar sobre la determinación de elementos traza en cervezas venezolanas por ICP-OES. Química Nova, v. 33, n. 3, p. 653-655, 2010. http://dx.doi.org/10.1590/S0100-40422010000300032.

MARDEGAN, S. F.; ANDRADE, T. M. B.; SOUSA NETO, E. R.; VASCONCELLOS, E. B. C.; MARTINS, L. F. B.; MENDONÇA, T. G.; MARTINELLI, L. A. Stable carbon isotopic composition of Brazilian beers: a comparison between large- and small-scale breweries. Journal of Food Composition and Analysis, v. 29, n. 1, p. 52-57, 2013. http://dx.doi.org/10.1016/j.jfca.2012.10.004.

MATSUSHIGE, I.; OLIVEIRA, E. Determination of trace elements in Brazilian beers by ICP-AES. Food Chemistry, v. 47, n. 2, p. 205-207, 1993. http://dx.doi.org/10.1016/0308-8146(93)90245-B.

MATUSIEWICZ, H.; KOPRAS, M. Methods for improving the sensitivity in atom trapping flame atomic absorption spectrometry: analytical scheme for the direct determination of trace elements in beer. Journal of Analytical Atomic Spectrometry, v. 12, n. 11, p. 1287-1291, 1997. http://dx.doi.org/10.1039/a704407k.

MENA, C.; CABRERA, C.; LORENZO, M. L.; LÓPEZ, M. C. Cadmium levels in wine, beer and other alcoholic beverages: possible sources of contamination. The Science of the Total Environment, v. 181, n. 3, p. 201-208, 1996. PMid:8820435. http://dx.doi.org/10.1016/0048-9697(95)05010-8.

MOORE, G. L. Introduction to inductively coupled plasma atomic emission spectrometry. Amsterdam: Elsevier, 1989. 340 p. v. 3.

MOURA-NUNES, N.; BRITO, T. C.; FONSECA, N. D.; AGUIAR, P. F.; MONTEIRO, M.; PERRONE, D.; TORRES, A. G. Phenolic compounds of Brazilian beers from different types and styles and application of chemometrics for modeling antioxidant capacity. Food Chemistry, v. 199, p. 105-113, 2016. PMid:26775950. http://dx.doi.org/10.1016/j.foodchem.2015.11.133.

NANO, R. M. W.; BRUNS, R. E.; FERREIRA, S. L. C.; BACCAN, N.; CADORE, S. Statistical mixture design development of digestion methods for Oyster tissue using inductively coupled plasma optical emission spectrometry for the determination of metallic ions. Talanta, v. 80, n. 2, p. 559-564, 2009. PMid:19836520. http://dx.doi.org/10.1016/j.talanta.2009.07.025.

OLIVEIRA, R. M.; ANTUNES, A. C. N.; VIEIRA, M. A.; MEDINA, A. L.; RIBEIRO, A. S. Evaluation of sample preparation methods for the determination of $\mathrm{As}, \mathrm{Cd}, \mathrm{Pb}$, and $\mathrm{Se}$ in rice samples by GF AAS. Microchemical Journal, v. 124, p. 402-409, 2016. http:// dx.doi.org/10.1016/j.microc.2015.09.018.

OÑATE-JAÉN, A.; BELLIDO-MILLA, D.; HERNÁNDEZ-ARTIGA, M. P. Spectrophotometric methods to differentiate beers and evaluate beer ageing. Food Chemistry, v. 97, n. 2, p. 361-369, 2006. http://dx.doi.org/10.1016/j.foodchem.2005.05.010.

ONIANWA, P.; ADETOLA, I.; IWEGBUE, C.; OJO, M.; TELLA, O. Trace heavy metals composition of some Nigerian beverages and food drinks. Food Chemistry, v. 66, n. 3, p. 275-279, 1999. http://dx.doi.org/10.1016/S0308-8146(98)00257-X.

ORESTE, E. Q.; JESUS, A.; OLIVEIRA, R. M.; SILVA, M. M.; VIEIRA, M. A.; RIBEIRO, A. S. New design of cold finger for sample preparation in open system: determination of $\mathrm{Hg}$ in biological samples by CV-AAS. Microchemical Journal, v. 109, p. 5-9, 2013. http://dx.doi.org/10.1016/j.microc.2012.05.034.

PALMER, J. J. How to brew: everything you need to know to brew beer right the first time. Boulder: Brewers Publications, 2006.

PASSAGHE, P.; BERTOLI, S.; TUBARO, F.; BUIATTI, S. Monitoring of some selected heavy metals throughout the brewing process of craft beers by inductively coupled plasma mass spectrometry. European Food Research and Technology, v. 241, n. 2, p. 199-215, 2015. http://dx.doi.org/10.1007/s00217-015-2445-7.

PINHEIRO, A. C. A.; LISBOA, M. T.; RIBEIRO, A. S.; NUNES, A. M.; YAMASAKI, A. Avaliação da mineralização de arroz em sistema de refluxo para a determinação de $\mathrm{Cu}, \mathrm{Fe}, \mathrm{Mn}$ e Zn por 
Decomposição de amostras de cerveja com sistema de refluxo para determinação monoelementar por $F$ AAS/AES e determinação multielementar por MIP OES

Leão, P. R. P. et al.

FAAS. Química Nova, v. 37, n. 1, p. 6-9, 2014. http://dx.doi. org/10.1590/S0100-40422014000100002.

POHL, P. Determination and fractionation of metals in beer: a review. Food Additives and Contaminants, v. 25, n. 6, p. 693-703, 2008. PMid:18484298. http://dx.doi.org/10.1080/02652030701772323.

POHL, P.; PRUSISZ, B. Fractionation analysis of manganese and zinc in beers by means of two sorbent column system and flame atomic absorption spectrometry. Talanta, v. 71, n. 4, p. 1616-1623, 2007. PMid:19071499. http://dx.doi.org/10.1016/j. talanta.2006.07.039.

ROSA, M. B.; ORESTE, E. Q.; BÖNEMANN, D. H.; RODRIGUES, A. A.; VENDRUSCOLO, C. T.; MOREIRA, A. S.; RIBEIRO, A. S.; NUNES, A. M. Evaluation of the use of a reflux system for sample preparation of xanthan gum and subsequent determination of $\mathrm{Ca}, \mathrm{Cu}, \mathrm{K}, \mathrm{Mg}, \mathrm{Na}$ and $\mathrm{Zn}$ by atomic spectrometry techniques. Journal of the Brazilian Chemical Society, v. 27, p. 919-924, 2016.
WIECZOREK, M.; RENGEVICOVA, S.; ŚWIT, P.; WOŹNIAKIEWICZ, A.; KOZAK, J.; KOŚCIELNIAK, P. New approach to H-point standard addition method for detection and elimination of unspecific interferences in samples with unknown matrix. Talanta, v. 170, p. 165-172, 2017. PMid:28501154. http://dx.doi.org/10.1016/j. talanta.2017.03.101.

WYRZYKOWSKA, B.; SZYMCZYK, K.; ICHICHASHI, H.; FALANDYSZ, J.; SKWARZEC, B.; YAMASAKI, S. Application of ICP sector field MS and principal component analysis for studying interdependences among 23 trace elements in Polish beers. Journal of Agricultural and Food Chemistry, v. 49, n. 7 , p. 3425-3431, 2001. PMid:11453786. http://dx.doi.org/10.1021/ jf010184g.

ZUFALL, C.; TYRELL, T. The influence of heavy metals ions on beer flavour stability. Journal of the Institute of Brewing, v. 114, n. 2, p. 134-142, 2008. http://dx.doi.org/10.1002/j.2050-0416.2008. tb00318.x. 